\title{
Synthesis of Vector Control Systems for Induction Motor Drives
}

\author{
Kucher Ekaterina S. \\ Department of Electric Drives and Industry Automation \\ Novosibirsk State Technical University \\ Novosibirsk, Russian Federation \\ kucher@corp.nstu.ru
}

\author{
Komazenko Maria A. \\ Department of Electric Drives and Industry Automation \\ Novosibirsk State Technical University \\ Novosibirsk, Russian Federation
}

\author{
Romashchenko Anastasia I. \\ Department of Electric Drives and Industry Automation \\ Novosibirsk State Technical University \\ Novosibirsk, Russian Federation
}

\begin{abstract}
This paper presents the methods of synthesis for speed systems control of induction motor drives, which in accordance with the principles of vector control are represented as a dynamic multi-input/multi-output object. Under the influence of uncontrolled signal and parametric disturbances, the synthesized speed control systems should ensure the proximity of processes to the desired, as well as the static accuracy of performance. Results can be obtained using special methods of structural and parametrical synthesis, such as methods of localization and high gains, which allow one to purposefully organize slow and fast movement loops, by establishing deep feedbacks, thereby ensuring a low sensitivity of the speed control system of induction motor drives to the influence of perturbations of various kinds.
\end{abstract}

Keywords - induction motor drive, slow movement loop, fast movement loop, high gains method, localization method.

The induction motor based on full digital control has reached the status of a mature technology. The world market volume is about 12000 millions US dollars with an annual growth rate of $15 \%$.

Ongoing research has focused on the elimination of the speed sensor at the machine shaft without deteriorating the dynamic performance of the drive control system [1]. Speed estimation is an issue of particular interest with induction motor drives where the mechanical speed of the rotor is generally different from the speed of the revolving magnetic field. The advantages of speed sensorless induction motor drives are reduced hardware complexity and lower cost, the reduced size of the drive machine, elimination of the sensor cable, better noise immunity, increased reliability and less maintenance requirements. The operation in hostile environments mostly requires a motor without a speed sensor.

Modern control techniques for ac motor drives were developed largely as a result of the search for low-cost alternative to high-performance four-quadrant dc drives. The use of shaft-mounted tacho generators' grids is established by practice. And the digital shaft-position encoder used in the mast effective vector-control schemes is considered acceptable. However, the sensor creates problems. Delicate optical encoders with internal signal-conditioning electronics are widely used. They lower the system reliability, especially in hostile environments, and require careful cabling arrangements with special attention to electrical noise. There are also situations where the positional feedback is extremely difficult to obtain. This is particularly true for the use of linear-motor drives. [1]

Until recently, the rapid developments in vector control technology have had little impact on adjustable-speed ac drives. They are typically impel voltage-source inverters with variable output frequency and are used for applications requiring little dynamic control. It has now become clear that these drives can benefit from the closed-loop current-control techniques that have evolved for use in vector control systems. Current control is readily applicable to existing voltage-source inverters, where it reduces the incidence of over-current tripping and improves inverter utilization.

Once the current converter is controlled, additional control must be provided to specify the magnitude and slip frequency of the ejected current vector, and, hence, to regulate the flux and torque of the motor. Motor speed feedback is typically required for outer-loop speed control as well as in the flux and torque control algorithm. This presents a problem in a lowperformance system where motor speed transducers are not usually available. This has led to renewed interests in "tacholess" vector control with the objective of providing an intermediate class of ac drives with enhanced performance and a wider range of applications than adjustable-speed drives but at about the same cost except for the small additional cost of more sophisticated control algorithms.

Great attention is paid to construction of control systems, taking into account the importance of factors of uncertainty, which include inconsistencies in the aprioristic information on values of parameters for the model, influence of external 
indignations. Also it is necessary to consider incompleteness of the information by a current condition of the object.

The greatest efficiency of stabilization of control of nonstationary objects is provided with the techniques based on a principle of deep feedback which allow setting not only static accuracy, but also desirable indicators of quality for transients of the control system. In such methods, it will be purposeful to organize slow and rapid movements of processes by means of which, after carrying out certain transformations, it is possible to isolate them into separate contours.

The contour of fast movements localizes both external and parametrical indignations - properties of object, and is the most internal contour of the control system.

Slow movements fully meet the requirements, and synthesis of the contour is carried out implicitly when choosing parameters of the equation of desirable movements. This contour provides for a small sensitivity to changes in the parameters of the object.

When constructing a control system by using the principle of separation of motion, it is possible to decompose the synthesis problems of the contours considered above, thereby simplifying the analysis of the solution - the contours of slow and fast movements can be synthesized separately from each other.

As a two-channel non-stationary object, the model of an asynchronous motor in a fixed coordinate system will be considered. It gives the chance to operate independently target variables of the object, i.e. a magnetic condition and the electromagnetic moment of the induction motor drive.

This paper presents a structural and parametrical synthesis of control systems of induction motor drives using the localization method and the high gains method. It also analyzes the results of studies of organized control systems, obtained with the help of digital simulation.

\section{MATHEMATICAL DESCRIPTION OF OBJECT}

The method frequency control of motor drives, which has received the name of a method of Field Oriented Control, allows one to present vectors of sinusoidally varying in time variables of induction motor changing with short-circuited rotor vectors of constant values. Therefore, analysis and synthesis of induction motor drives of control systems has become easier.

The use of spatial vectors as complex state variables is an efficient method for ac machine modelling. Vector controlled drives require estimating the magnitude and spatial orientation of the fundamental magnetic flux waves in the stator or in the rotor. They differ with respect to accuracy, robustness, and sensitivity to variations in model parameters. Dynamic performance and steady-state speed accuracy in the low speed range can be achieved by exploiting parasitic effects of the machine. [4]

The space vector approach represents the induction motor as a dynamic system of only a third order, and permits an insightful visualization of the machine and the superimposed control structures by complex signal flow graphs [3]. The approach implies that the spatial distributions along the airgap of the magnetic flux density, the flux linkages and the current densities (magnetomotive force, mmf) are sinusoidal. Linear magnetics are assumed while iron losses, slotting effects, deep bar and end effects are neglected

To describe the concept of a spatial vector, a three-phase stator winding is used. The winding axis of the phase is aligned with the real axis of the complex plane. To create a sinusoidal flux density distribution, the stator of magnetomotive force (MMF) must be a sinusoidal function of the circumferential coordinate. The distributed phase windings of the machine model are therefore assumed to have sinusoidal winding densities. Then each phase current creates a specific sinusoidal MMF distribution, the amplitude of which is proportional to the respective current magnitude, while its spatial orientation is determined by the direction of the respective phase axis and the current polarity.

During a steady-state operation, the stator phase currents form a balanced, sinusoidal three-phase system, which cause the stator MMF to rotate at constant amplitude with synchronization of the angular frequency of the stator currents. [4]

The principle of space vector control is based on the mathematical model of the ac motor which allows presenting the engine as a two-channel object of control in the field oriented orthogonally the system of coordinates on a vector of the flux linkage. It gives the chance to operate independently variables of the object, i.e. a magnetic condition and the electromagnetic moment of the ac machine.

Let us consider the mathematical description of the ac engine in the field oriented system of coordinates $(d, q)$ rotated relatively motionless coordinate system $(\alpha, \beta)$ at such angle $\gamma_{\psi}$ at which longitudinal axis $d$ coincides in the direction with the vector of flux linkage $\Psi_{r}$. When using system $(d, q)$, the cyclic speed of rotating system coordinates is equal to instant speed of a vector of flux linkage rotor $\omega_{k}=\omega_{\psi}$, and the equations of the induction motor (1) in the scalar form of the recording take the form [1, 2, 3, 4]

$$
\left.\begin{array}{c}
L_{\sigma e} \cdot \frac{d i_{s d}}{d t}=-R_{s} \cdot i_{s d}-\frac{L_{m}}{L_{r}} \cdot \frac{d \psi_{r m}}{d t}+\omega_{\psi} \cdot L_{\sigma e} \cdot i_{s q}+u_{s d}, \\
L_{\sigma e} \cdot \frac{d i_{s q}}{d t}=-R_{s} \cdot i_{s q}-\omega_{\psi} \cdot\left(L_{\sigma e} \cdot i_{s d}+\frac{L_{m}}{L_{r}} \cdot \psi_{r m}\right)+u_{s q}, \\
\frac{d \psi_{r m}}{d t}=\frac{L_{m}}{T_{r}} \cdot i_{s d}-\frac{1}{T_{r}} \cdot \psi_{r m}, \\
\frac{d \gamma_{\psi}}{d t}=\omega_{\psi}, \\
M_{e}=p_{n} \cdot \frac{L_{m}}{L_{r}} \cdot \psi_{r m} \cdot i_{s q},
\end{array}\right\}
$$

where $\psi_{r m}, \omega_{\psi}$ - Euclidean norm and instantaneous circular frequency of the rotor flux linkage vector; $i_{s d}, i_{s q}-$ magnetizing and torque-forming components of the stator 
currents vector; $u_{s d}, u_{s q}$ - components of the stator voltage vector; $\omega_{s}$ - rotor slip frequency; $\omega_{k}$ - frequency of $\mathrm{k}$ coordinates; $\omega_{e}$ - electric rotor speed; $D$ - rotation matrix; $L_{r}, L_{m} . R_{r}, R_{s}, L_{\sigma e}-$ parameters of the AC engine ; $T_{r}=\frac{L_{r}}{R_{r}}$ - rotor time constant of the induction machine, $L_{\sigma e}-$ equivalent motor leakage inductance.

For completeness of the mathematical description of the $\mathrm{AC}$ machine, it is also necessary to consider the equation of movement of the electric motor drive:

$$
J \cdot \dot{\omega}=M_{e}-M_{c},
$$

where $M_{e}$ - electromagnetic torque, $J$ - total moment of inertia; $M_{c}-$ load torque, $p_{n}$ - number of pole pairs.

The principle of vector space control allows one to influence independently $i_{s d}$ - magnetizing and $i_{s q}$ - torqueforming components of the stator currents vector for control magnetic condition of the AC machine and the electromagnetic moment accordingly, as in the direct current engine.

\section{STRUCTURAL SYNTHESIS OF SPEED CONTROL SYSTEM OF INDUCTION MOTOR DRIVE}

Many schemes are based on the simplified engine models. They have been devised to sense the speed of the induction motor of the measured terminal quantities for control purposes. In order to obtain an accurate dynamic representation of the motor speed, it is necessary to base the calculation in the coupled circuit equation of the motor. [2]

Synthesis of the speed control system of the induction motor drive is carried out on the basis of the principle for subordinate regulation, then the structural scheme of the system vector control will look as shown in Fig. 1.

Most drive systems have a current control loop incorporated in their control structure. It is therefore advantageous to select the stator current vector as one state variable. The second state variable is a stator flux or rotor flux linkage vector, depending on the problem at hand. Selecting the rotor current vector as a state variable is not very practical, since the rotor currents cannot be measured in a squirrel cage rotor.

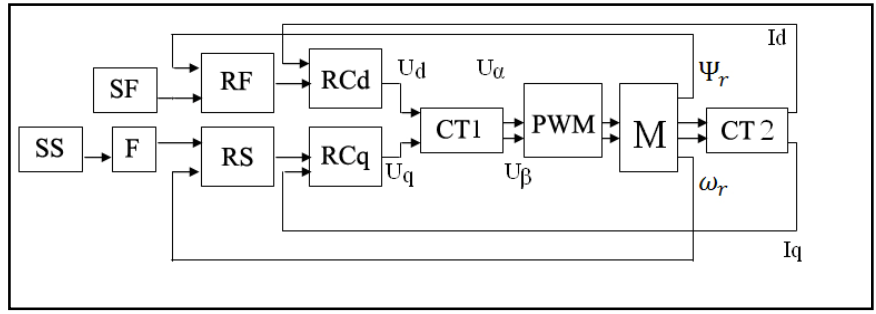

Fig. 1. Structural scheme of system vector control
Fig. 1 shows RF - flux linkage regulator, $\mathrm{RCd}$ - regulator of magnetizing component stator current, $\mathrm{RCq}$ - regulator of component stator current, $\mathrm{RS}$ - speed regulator, $\mathrm{F}$ - filter, $\mathrm{M}-$ AC machine, SF - setting signal in the flux linkage control channel, SS - setting signal in the speed control channel, CT1 - coordinate transformer, CT2 - coordinate transformer.

\section{SYNTHESIS OF SYSTEM CONTROL MOTOR DRIVE BY HIGH GAINS METHOD}

Regulators of the flux linkage rotor, speed and currents of the stator on longitudinal and cross axes are synthesized based on the astatic law for control of the system $[5,6,7]$

$$
u=\mu^{-1}\left(\varepsilon+\mu^{-1} c \int_{0}^{t} \varepsilon d t\right)
$$

where $u$ - setting influence; $\mu^{-1}-$ high gain of the regulator; $\varepsilon=i^{\text {ref }}-i$ - regulation error; $c$ - some constant coefficient, $\mu$-small parameter.

Using Laplace's transformation for equation (2), one will receive the transfer function of regulators, having an identical appearance for all regulators of system control

$$
W(p)=\frac{1}{\mu}+\frac{c}{\mu^{2}} \cdot \frac{1}{p} .
$$

With further parametric synthesis of control loops, assume that

$$
W_{R C}(p) \approx \frac{U_{3 \max }}{\lambda I_{H}}
$$

The characteristic polynomial of the closed system

$$
\begin{gathered}
D(p)=\frac{\mu^{2} K_{o c t} J}{c_{\kappa p c} c K_{r} \psi_{r m} K_{o c c}} p^{2}+\frac{\mu}{c_{\kappa p c} K_{o c s}} p+1, \\
D(p)=T_{\kappa p c}^{2} p^{2}+2 \xi_{\kappa p c} T_{\kappa p c} p+1
\end{gathered}
$$

where $c=p_{n} ; K_{r}=\frac{L_{m}}{L_{r}}, T_{\kappa p c}-$ time constant of the speed control loop, $\xi_{\kappa p c}$ - contour damping factor; $K_{o c t}, K_{o c s}-$ feedback coefficients of rotor speed and stator current control loops.

Parametric synthesis of the speed control loop consists in determining $c_{\kappa p c}$ and $\mu_{\kappa p c}$ for equation (3). Given there is speed and requirements for the process of transition of the control loops, let us determine the parameters of the speed controller:

$$
\begin{gathered}
c_{\kappa p c}=\frac{4 \cdot \xi_{\min }^{2} \cdot K_{o c t} \cdot J \cdot K_{o c s}}{c K_{r} \cdot \psi_{r m}}, \\
\mu_{\kappa p c}=T_{\kappa p c} \sqrt{\frac{c_{\kappa p c} \cdot c \cdot K_{r} \cdot \psi_{r m} \cdot K_{o c s}}{K_{o c t} \cdot J}} .
\end{gathered}
$$


Using the method described above, let us perform a parametric synthesis of the induction motor of the rotor flow regulator:

$$
c_{\kappa p n}=\frac{4 \cdot \xi_{\min }^{2} \cdot K_{o c t} \cdot T_{r} \cdot L_{m} \cdot K_{o c n}}{\left(L_{m} \cdot K_{o c n}+\mu_{\kappa p n} \cdot K_{o c t}\right)^{2}},
$$

where $\mu_{\kappa p n}=\frac{\mu_{\kappa p c}}{\eta}$ - small parameter of the rotor flux linkage regulator, $\eta$ - degree for separation of motion, $K_{o c n}$ - feedback coefficients of the induction motor of control loop of rotor flux linkage.

For control loops of currents on longitudinal and cross axes, coefficients $c_{\kappa p m}$ and $\mu_{\kappa p m}$ are:

$$
c_{\kappa p m}=\frac{4 \xi_{\min }^{2} K_{o c t} T_{\sigma} R_{S} K_{n}}{\left(K_{n} K_{o c t}+\mu_{\kappa p t} R_{s}\right)^{2}}, \mu_{\kappa p t}=\frac{\mu_{\kappa p n}}{\eta},
$$

where $K_{n}$ - transfer factor of the frequency converter; $T_{\sigma}=L_{\sigma e} / R_{S}-$ electromagnetic time constant.

For calculation of estimations for regulated variables and maintenance of necessary noise immunity of the system, the differentiating filter is used $[5,6,7]$ :

$$
T_{f}^{2} \widehat{I}_{s}^{(2)}+A_{1 f} T_{f} \widehat{I}_{s}^{(1)}+\widehat{I}_{s}=I_{s},
$$

where $T_{f}=\frac{\mu_{\kappa p m}}{\eta}-$ time constant of the differentiating filter; $A_{1 f}$ - characteristic polynomial coefficient, $\hat{I}_{S}-$ estimation of stator current.

The structural scheme of the system vector control synthesized by the high gains method is shown in Fig. 2, where the following notations are introduced: DFCd differentiating filter of stator current for the longitudinal axis; DFCq - differentiating filter of stator current for the cross axis.

In this control system, three rates of movement are organized, where there is the superfast rate of the movement differentiating filter

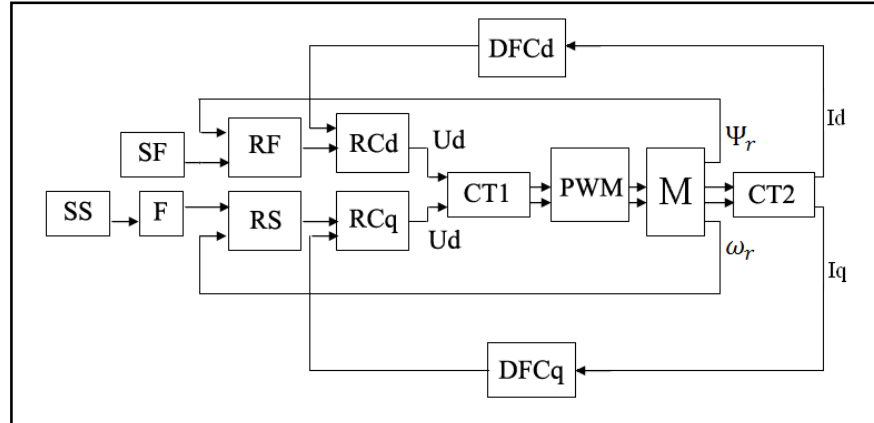

Fig. 2. Structural scheme of system vector control, synthesized by high gains method

\section{PARAMETRIC SYNTHESIS OF THE CONTROL SYSTEM OF INDUCTION MOTOR DRIVE BY THE LOCALIZATION METHOD}

To illustrate the method idea, let us assume that the state vector and the highest derivative $x^{(n)}$ of the controlled coordinate are accessible to direct measurements. The control error is given by $[8,9,10]$ :

$$
\varepsilon=x_{g}^{(n)}-x^{(n)}
$$

where $x_{g}^{(n)}=F(v, x)$ - equation of desired motion of the highest derivative controlled coordinate; $v(t)$ - setting influence.

The stator current control loop with a proportional-integral regulator on the longitudinal and cross axes describes the following system of equations $[5,6,8]$ :

$$
\left\{\begin{array}{c}
i_{s q}^{(1)}=K_{P} \mathcal{E}^{(1)}+K_{I} \varepsilon \\
i_{s q}^{(2)}+A_{1} \Omega_{g} i_{s q}^{(1)}+\Omega_{g}^{2} i_{s q}=\Omega_{g}^{2} U_{S C}, \\
T_{f}^{2} \widehat{i}_{s q}^{(2)}+A_{1 f} T_{f} \hat{i}_{s q}^{(1)}+\hat{i}_{s q}=i_{s q} .
\end{array}\right.
$$

The first equation of system (5) is the control law, where $K_{P}, K_{I}-$ coefficients of the proportional and integral regulator part. The following equation is the system equations of desired motions, where $A_{1}$ - coefficient of desired motion; $\Omega_{g}$ - compound root of desired motion; $U_{S C}$ - setting influence of stator current. The third equation of system (5) is the mathematical description of the differentiating filter.

Let us obtain the equation of stator current error from the equation of desired motion:

$$
\begin{gathered}
i_{s q}^{(2)}=\Omega_{g}^{2} U_{S C}-A_{1} \Omega_{g} i_{s q}^{(1)}-\Omega_{g}^{2} i_{s q}, \\
\varepsilon=i_{s q}^{(2)}-\widehat{i}_{s q}^{(2)} .
\end{gathered}
$$

To find the coefficients of the proportional and integral regulator part, let us make the structural synthesis of the stator current control loop in accordance with the system of equations (5) and (6) (Fig. 3).

$$
\text { Let us accept that } \frac{K_{n}}{\tau_{\mu} p+1} \approx K_{n}, D_{f}(p) \approx T_{f} p+1 \text {. }
$$

Then the characteristic equation of fast movement of the inner contour (Fig. 3) is equated to the normalized characteristic equation of the fast motions subsystem, which has the following form $[5,6,8]$ :

$$
T_{b}^{3} p^{3}+T_{b}^{2} A_{2 b} p^{2}+T_{b} A_{1 b} p+1=0,
$$

where $T_{b} \leq \frac{T_{g}}{\eta}$ - time constant of the fast movement loop, $T_{g}=\frac{1}{\Omega_{g}}-$ time constant of desired motions; $A_{i b}-$ characteristic polynomial coefficient. 


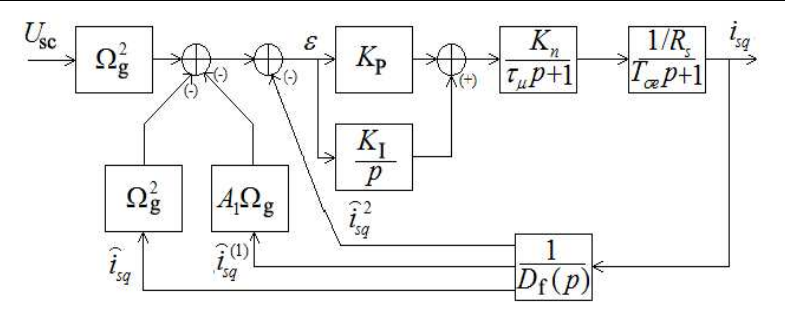

Fig. 3. Structural scheme of the stator current control loop

Taking into account the value of the time differentiation filter, the degree for separation of motion and the coefficients of the characteristic polynomial fastmovementloop is calculated as the coefficients of the proportional and integral part of the current regulator:

$$
K_{I}=\frac{R_{s} T_{f} T_{\sigma e} \Omega_{g}{ }^{3} \eta^{3}}{K_{n}}, K_{P}=\frac{T_{b} A_{1 b} K_{I} K_{n}-R_{s}}{K_{n}} .
$$

The regulation loop of the rotor flux-linkage with the proportional-integral regulator is also described by three equations:

$$
\left\{\begin{array}{c}
U_{s d}^{(1)}=K_{P} \varepsilon^{(1)}+K_{I} \varepsilon \\
\psi_{r}^{(2)}+A_{1} \Omega_{g} \psi_{r}^{(1)}+\Omega_{g}^{2} \psi_{r}=\Omega_{g}^{2} U_{S F}, \\
T_{f}^{2} \widehat{\psi}_{r}^{2}+A_{1 f} T_{f} \widehat{\psi}_{r}^{(1)}+\widehat{\psi}_{r}=\psi_{r} .
\end{array}\right.
$$

where $U_{S F}$ - setting influence of the rotor flux linkage; $\widehat{\psi}_{r}-$ estimation of the rotor flux linkage.

As well as in the stator current loop, the equation for the error in regulation of the rotor flux linkage is obtained from the equation of desired motions:

$$
\varepsilon=F(v, x)-\widehat{\psi}_{r}^{(2)}
$$

Using the system of equations (8) and (9), let us construct the structural scheme of the control loop of rotor flux and find the coefficients of the flux proportional-integral regulator (Fig. $4)$.

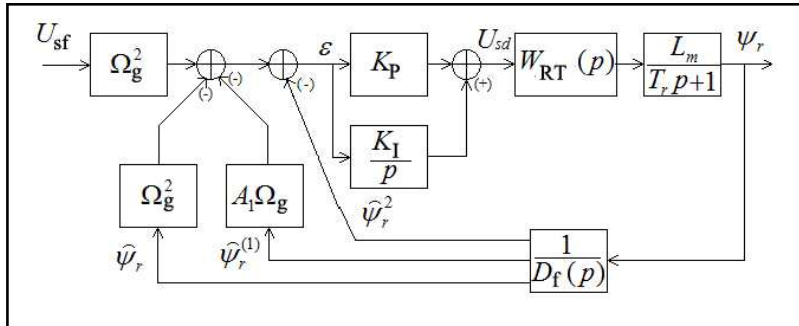

Fig. 4. Structural scheme of the rotor flux control loop

Let us assume that the transfer function of the closed loop current control will be determined as follows:

$$
W_{R C}(p) \approx \frac{1}{K_{o c t}}
$$

Further, according to the method described above, the characteristic polynomial of the inner contour of movements (Fig. 4) is equated to the normalized polynomial of the form (7) and the coefficients of the proportional-integral regulator of the flux linkage is calculated, while it is necessary to set the filter time constant, the degree for separation of motion and the normalized polynomial coefficients of the fast movement loop:

$$
K_{I}=\frac{T_{r} T_{f} K_{o c t} \Omega_{g}{ }^{3} \eta^{3}}{L_{m}}, K_{P}=\frac{T_{b} A_{1 b} K_{I} L_{m}-K_{o c t}}{L_{m}} .
$$

When synthesizing the rotor speed control loop, it is necessary to specify the same three equations:

$$
\left\{\begin{array}{c}
U_{s q}^{(1)}=K_{P} \varepsilon^{(1)}+K_{I} \varepsilon, \\
\omega_{r}^{(2)}+A_{1} \Omega_{g} \omega_{r}^{(1)}+\Omega_{g}^{2} \omega_{r}=\Omega_{g}^{2} U_{S C}, \\
T_{f}^{2} \widehat{\omega}_{r}^{2}+A_{1 f} T_{f} \widehat{\omega}_{r}^{(1)}+\widehat{\omega}_{r}=\omega_{r},
\end{array}\right.
$$

where $U_{S C}-$ setting influence of the speed rotor; $\widehat{\omega}_{r}-$ estimation of the speed rotor.

The expression for determining the speed control error will have the form:

$$
\varepsilon=F(v, x)-\widehat{\omega}_{r}^{(2)} .
$$

Using the system of equation (10) and expression (11), let us make structural synthesis of the rotor speed control loop of the induction motor drive (Fig. 5).

Parametric synthesis of the contour under consideration is carried out according to the method described above, which was applicable for determining parameters of the stator current control loops and the rotor flux linkage coupling of the asynchronous motor.

Expressions determine the speed regulator coefficients of the proportional and integral part

$$
K_{I}=\frac{T_{r} K_{o c t} J \Omega_{g}{ }^{3} \eta^{3}}{\psi_{r} c K_{r}}, K_{P}=T_{b} A_{1 b} K_{I}
$$

The structural scheme of the electric motor drive of the vector control system, synthesized by the localization method, is analogous to the structural scheme of the control system, synthesized by the high gains method. (Fig. 2).

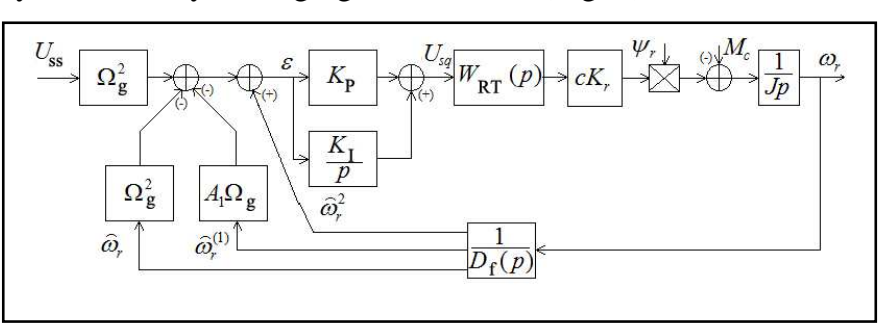

Fig. 5. Structural scheme of rotor speed control loop 


\section{RESEARCH RESULTS}

The correctness of structural and parametric synthesis and the operability of vector control systems of the induction motor drive were confirmed by digital simulation in software package MATLAB Simulink. Structural and parametric synthesis of control systems that are insensitive to changes in the induction motor drive parameters was carried out for a general industrial induction motor drive of medium power with different rotational rotor speeds.

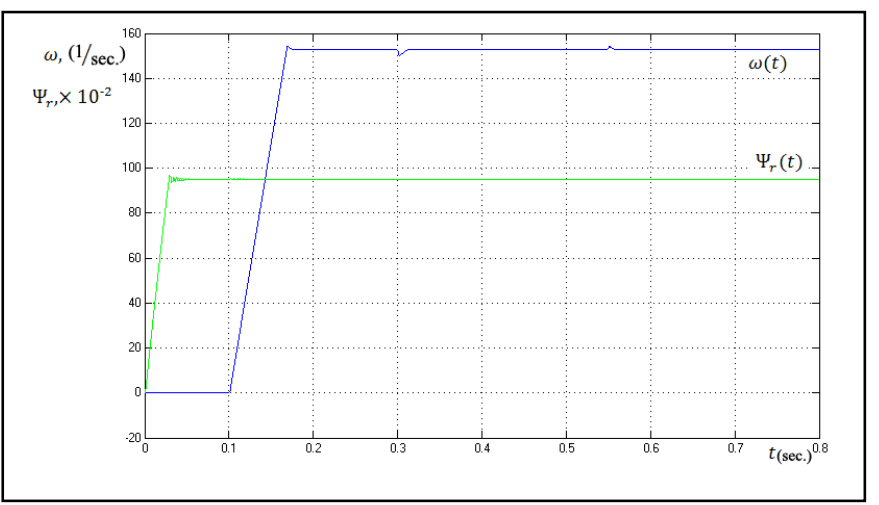

Fig. 6. Transition processes of speed and flux linkage of rotor for high gains method

The results of approbation for developed algorithms are graphs of the rotor speed transient processes and the rotor flux linkage module of the high gains method of asynchronous motors (Fig. 6) and the localization method (Fig. 7), which show that control systems provide the desired shape and parameters of quality of transient processes when introducing both parametric and signal disturbances.

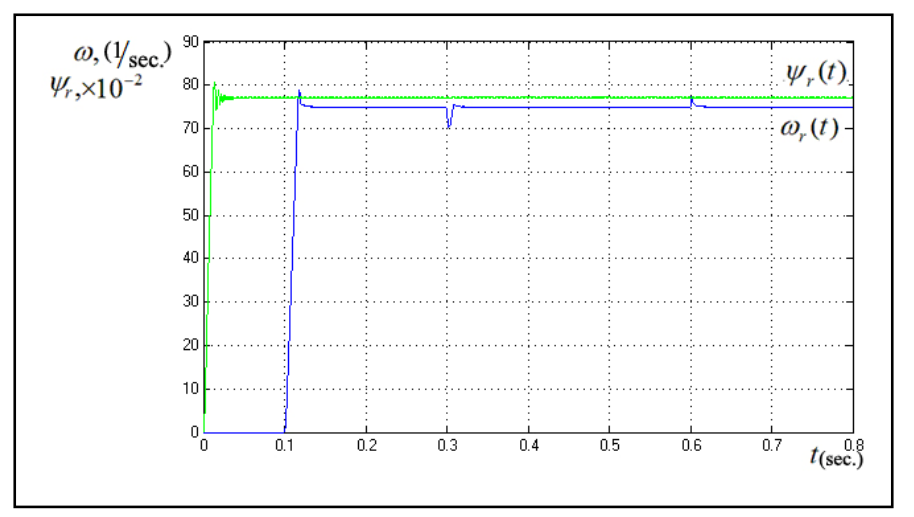

Fig. 7. Transition processes of speed and flux linkage of rotor

\section{CONCLUSION}

Proposed methods for synthesis of control systems make it possible to provide low sensitivity to introduction of parametric perturbations with the active resistance of the stator winding and the rotor winding of the induction motor drive.

Use of differentiating filters in the internal control of longitudinal and cross components of stator current, highfrequency noise can be eliminated in control systems that can be contained in sensor signals.

The establishment of "deep" feedbacks in synthesized control systems is possible for localized effect of changes in the electric motor drive parameters by (10-15)\% of nominal values. With the increase of changes in parametric disturbances, as well as the extended range of the regulation rotor speed of the induction motor drive, it is necessary to use special algorithms for parametric on-line identification and adaptation.

\section{References}

[1] K. Rajashekara, A. Kawamura, K. Matsuse, "Sensorless control of AC motor drives, speed and position sensorless operation, IEEE Press, pp. 1-17, 1996.

[2] X. Xu, D. Novotny, "Implementation of direct stator flux orientation control on a versatile DSP based system", IEEE Transactions on Industry Applications, Vol. 27, No 4, pp 694-700, 1991.

[3] J. Holtz, "Methods for speed sensorless control of AC drives", IEEE PCC-Yokohama, pp. 415 - 420, 1993.

[4] J. Holtz, "Sensorless Control of induction motor drives", IEEE, vol. 90, no. 8, pp. $1358-1394,2002$.

[5] V.V. Pankratov, Y.A. Zima, "Multi-objective optimization of fieldoriented control systems for induction motor electric drives", Proceedings of 1st Russian - Korean International Symposium on Applied Mechanics RUSKO-AM-2001, pp. 157 - 160, October 2001.

[6] V.V. Pankratov, Y.A. Zima, "The synthesis method of discrete controllers for nonlinear systems", Actual Problems of Electronics Instrument Engineering, Vol. 1,pp. 123 - 128, 2000.

[7] V.V. Pankratov, Y.A. Zima, "Synthesis of the discrete continuous systems by the high gain method", Automation, Control, and Information Technology, pp. 306 - 309, June 2002.

[8] Y.A. Zima, V.V. Pankratov, "Synthesis of astatic multivariable systems by the localization method", Actual Problems of Electronics Instrument Engineering, Vol. 1, pp. 236 - 240, 2002.

[9] S. Bolognani, L. Peretti, M. Zigliotto, "Parameter sensitivity analysis of an improved open-loop speed estimate for induction motor drives", IEEE Transactions on Power Electronics, Vol. 23, No. 4, pp. 2127 2135, July 2008.

[10] G. Henneberger, B.J. Brunsbach , Th. Klepsch, "Field-oriented control of synchronous and asynchronous drives without mechanical sensors using a Kalman filter", IEEE Press, pp. 207-213, 1996. 